\title{
Isolated Psychiatric Ward Patients in Southern Israel with Severe Mental IIInesses Describe Their Sexual Needs: A Qualitative Study
}

\author{
Michal Kaplan ${ }^{1} \cdot$ Tal Peleg-Sagy ${ }^{2,3}$ - Jonathan Guez ${ }^{2,4} \cdot$ Iftach Sagy $y^{3,5}$
}

Accepted: 19 December 2021 / Published online: 19 January 2022

(c) The Author(s) 2022

\begin{abstract}
Intimacy and sexuality are fundamental issues in human nature, but have been neglected in the rehabilitation of mental health patients, particularly those admitted into psychiatric wards. Patients' sexuality is excluded from the discourse; yet it exists in the daily lives of the patients. The study examines the sexual needs and challenges of people with severe mental illnesses admitted to an isolated psychiatric ward. 13 men were interviewed in a semi-structured interview regarding their sexuality and the challenges they meet when experiencing and expressing their sexuality as patients in an isolated ward. A thematic analysis was conducted. Two principal themes were identified: 1. Challenges in realizing sexuality within the isolated ward. This theme included sub themes: performing sexual acts and exposure to sexuality within the ward; medications' side effects; dialogue with the staff; sexuality in the ward presented as crossing limits; and absence of a space to fulfill sexuality. 2. Suggestions for improvements in the hospital, stemming from the patients' experiences and ideas, such as starting an open dialogue with the staff, supplying patients with measures for protected sex, and more. The findings highlight the lack of healthy expression of sexuality inside the wards, despite its importance for improving patients' well-being during their rehabilitation. Medical staff and policymakers should be aware of the sexual aspects of hospitalization in a psychiatric ward. The study also indicates the need for national policy that will address patients' sexual needs, including clear instructions that consider the uniqueness of the target population.
\end{abstract}

Keywords Severe mental illness $\cdot$ Sexuality $\cdot$ Isolated psychiatric wards $\cdot$ Israel $\cdot$ Medical staff · Rehabilitation $\cdot$ Qualitative research

\section{Background}

A severe mental illness (SMI) is defined as a mental, behavioral, or emotional disorder resulting in serious functional impairment, which substantially interferes with or limits one or more major life activities. These illnesses include disorders that produce psychotic

Michal Kaplan

kapmicha1951@gmail.com

Extended author information available on the last page of the article 
symptoms, such as schizophrenia, and severe forms of other disorders, such as major depression and bipolar disorder [29]. According to the Substance Abuse and Mental Health Services Administration [36], mental illnesses are prevalent in the United States: in 2019, about 13.1 million adults older than 18 (5.2\%) were diagnosed with SMI. Among them, $65.5 \%$ (or 8.6 million people) received mental health services. Similar rates were recorded yearly between 2008 and 2018 .

According to the Mental Health Division of Israel's Ministry of Health [6], Israel had 18,096 inpatients hospitalized in 2019, and the age adjusted rate was 2.03 per 1,000 persons. There were 6.1 times more male than female inpatients. The rate of inpatients returning within 30 days was 19\%. Out of all patients in psychiatric hospitals in Israel, $47 \%$ are people with schizophrenia. Most of these cases are re-hospitalizations.

Sexuality is a physiological human necessity, which joins other physiological needssuch as eating, drinking, and sleep - to form the base of Maslow's hierarchy of needs [20, 24]. In a psychiatric ward, even when sexuality is denied or labeled as 'dangerous' by the medical staff, it continues to secretly exist. As we can learn from the study of Buckley et al. [11], even in a hospital where sexual intercourse was completely prohibited, half of the patients who participated in the study did have sexual contacts. Patients continue to masturbate, have sexual contact with other patients, and have sexual wishes and drives. This reality transforms sexuality from a healthy need that exists within legitimate boundaries to an unsupervised behavior that is expressed in inappropriate settings.

According to a common assumption, SMI patients do not need to have a sexual life. This, however, is a misguided belief. Patients avoid speaking to the medical staff about their sexuality, leading to unfulfilled sexual needs, which may in turn cause risky sexual behavior (such as prostitution or unprotected sex). These aspects of mental health treatment are particularly significant since psychiatric patients suffer from increased rates of sexual functioning disorder, which harms their life quality. Thus, it is important that the treatment of psychiatric patients include elements that address their sexuality, allowing them to maintain sexual health and promoting their life quality [20]. Considering the goal of mental health services to provide holistic care, it is important to take an approach that adjusts to the needs of each patient's rehabilitation process and consider the various aspects of care that may improve his or her life [5]. Empirical evidence points to the potential of sexual well-being to promote patients' rehabilitation and healing and increase positive behavioral motivation, thereby assisting in their emotional and psychological treatment [8]. According to Cook [12], the expression of healthy sexuality increases well-being among SMI patients. The realization of sexuality enhances patients' quality of life and self-esteem. Moreover, healthy sexual relationships may assist patients in overcoming both their social and their sexual isolation [3].

According to federal law, patients with SMI who live in long-term psychiatric facilities have sexual rights. The law grants them the right for privacy for intimate relationships [30]. Sexual rights are grounded in universal human rights. Realizing sexual rights allows realization of the rights for autonomy, physical integrity, and freedom of expression [18]. Social and cultural taboos around sexuality often affect legislation and lead to policies that compromise sexual health [18]. The World Health Organization [40] claims that patients' sexual rights must be respected and allowed to be fully implemented. The current literature also maintains that hospitals should allow sexuality to exist while protecting patients' safety and acknowledging the tension between the right for safety and the right for sexuality, autonomy, and respect. There are potential risks both to providing opportunities for sexual activity in the hospital and to coerced sexual activity or control over another person. Therefore, hospitals must establish clear guidelines for maintaining patients' health [25]. 
Despite the importance of the topic, sexuality is insufficiently addressed within clinical wards, among other reasons due to a common preoccupation with the disease's symptoms and effects [35]. Acknowledging the patients' sexual rights can play an important role in promoting sexual health and well-being and is a necessary condition for sustained social and economic development of communities and nations [22]. People's right for enjoyable and safe sexual experiences - free of discrimination, coercion, or violence-is fundamental to the health and well-being of humankind.

A variety of obstacles may account for the gap between the healthy need for sexuality and its expression in the ward. For many patients, impaired sexual function results from the side effects of psychiatric medication. Many such medications severely affect patients' arousal, desire, and orgasm [38]. This phenomenon is more common in men, and the functional impairment varies according to the type of medication [7]. Also, Cook [12] suggests that some of the barriers are related, among other things, to the lack of privacy in the wards, to a history of sexual abuse, and to the stigmas that accompany mental disease, which in turn make it challenging to establish emotional relationships. Another reason for this gap is that caregivers find it difficult to discuss the subject with the patients, since they do not receive appropriate training to approach these issues [13]. The topic of sexuality is scarcely discussed among the medical teams, who exhibit reduced involvement in their patients' sexual needs compared to other aspects of their health [33]. The literature also reveals that the sexual needs of psychiatric patients with criminal background in long term hospitalization receive little attention [39]. All in all, the result is that psychiatric patients remain with inadequate solutions for their sexual needs [13].

Within the policy that developed over the years, emphasis has been placed on allowing patients to express healthy sexuality under certain limitations [28]. Buckley and Hyde [9] found that $83 \%$ of the hospitals included in their study had sexual behavior policies and $75 \%$ of those facilities had sexual education programs. Still, federal regulations were ignored by the staff [10]. Research also shows that in recent years, reports by medical teams have lacked data on patients' sexual behavior, indicating that the staff does not acknowledge the sexuality of mental patients. These findings emphasize the need to promote the education of mental health professionals about patients' sexuality [16].

A study that examined policy regarding expression of sexual needs among psychiatric patients in 14 European Union counties found that none of the countries had consistent national policy that considered patients' sexual needs [39]. The current literature presents similar findings from various countries, indicating the absence of official policy $[32,34]$. Israel too lacks such national policy. A review of Israeli policy papers did not identify any written guidelines on the topic. Thus, it seems that there is currently no consistent and comprehensive policy that dictates sexuality-related practice within hospitals in Israel. Taken together, the current literature confirms that the existing approach to mental patients' sexuality is inadequate and requires reexamination [25]. New, progressive policy is needed to truly address patients' sexual needs and go beyond perceiving the issue in terms of risk management [39]. Although the problem is well known, there is only scarce research that examines the current situation in isolated wards with an emphasis on the needs and challenges as seen through the patients' eyes.

In the current study, the researched population is uniquely comprised of men who have been diagnosed with SMI and hospitalized in a closed ward. The purpose of this study is to investigate how these isolated psychiatric ward patients describe their sexual needs, based on their personal experiences inside the ward, the challenges they meet, and the solutions they find. There are expected gaps between the existence of sexual needs and the absence of an actual response from the therapeutic staff. Hearing the voices of the patients 
may ameliorate sexual expression and allow healthy sexuality to exist, which may improve patients' well-being and assist in their rehabilitation and integration into normative life.

Few papers about psychiatric inpatients have given a voice to the patients themselves and allowed them to express their personal hospitalization experiences and suggest changes. This study is further innovative in putting the spotlight on a population that has not previously been reported: occupants of a closed psychiatric ward in Israel, including Jewish and Arab patients.

The ward is located in southern Israel, a region considered as geographic and socioeconomic periphery. Periphery is a concept with both geographic and social meanings. Geographic periphery is defined by its geographic location and distance from central Israel. The Israeli geographic periphery is made up of two districts: northern Israel and southern Israel. The term social periphery refers to socio-economically disadvantaged communities [21]. There is evidence of gaps between central Israel and peripheral regions in various life aspects: income, health, opportunity for quality education, and more [31], 37.

The participants in this study include Bedouin Arabs, who are a subgroup within the Arab minority in Israel and have unique culture, history, social and political characteristics [23]. The Bedouins are Muslim, with a traditional-collectivist way of life. They are the group with the lowest socioeconomic status in Israel [1]. Mental health systems in Israel are perceived by Arabs as representing Western values and ignoring Muslim traditions [2]. The special ethnic and peripheral characteristics of the target population add an interesting perspective to the research, especially in comparison to studied populations in Europe and the United States. The present study does not provide a comparison between Arabs and Jewish patients or between them and other groups, but offers a glimpse into the sexual needs of this unique population.

\section{Methods}

\section{Participants}

Thirteen men hospitalized in an isolated ward for men within a psychiatric hospital in the southern district of Israel participated in the study. Participants had been diagnosed with schizophrenia, psychotic disorders, or bipolar disorder (according to DSM-V-TR criteria). Participants were approached in a sensitive manner, employing careful consideration regarding the patient's mental state, his stability, and his ability to answer questions about sexuality. The sampling is a convenience-sampling type, in which all patients in the ward who met the following criteria and were willing to participate were sampled. All participants were above 18 years old, classified by the medical staff as being in partial remission, with satisfactory discretion to give participation consent, and demonstrating no dangerous behavior or an eroto-manic psychotic component. Various demographic details were collected through a demographic characteristic questionnaire relating to age, religion, country of birth, marital status, etc. Ten of the participants were Jewish and three were Arab, aged 32-67 $(\mathrm{M}=46.03)$. Eleven participants were single or divorced, and two were married. Only three of the unmarried participants were in a relationship at the time of the study. Participants' demographic characteristics are presented in Table 1.

Exclusion Criteria Patients were excluded from the study in the following cases: first, if a participant expressed distress in response to the study materials. Second, if the participant's interviewer/physician noticed that the interview caused distress or dangerous 
Table 1 Socio-demographic characteristics

\begin{tabular}{|c|c|c|c|c|}
\hline & $\mathrm{n}$ & $(\%)$ & M & SD \\
\hline Age (years) & & & 46.03 & 12.12 \\
\hline $32-46$ & 8 & $(61.54)$ & & \\
\hline $47-61$ & 3 & $(23.08)$ & & \\
\hline $62-67$ & 2 & $(15.38)$ & & \\
\hline \multicolumn{5}{|l|}{ Place of birth } \\
\hline Native-born & 8 & $(61.54)$ & & \\
\hline Foreign-born & 5 & $(38.46)$ & & \\
\hline \multicolumn{5}{|l|}{ Nationality } \\
\hline Jewish & 10 & (76.92) & & \\
\hline Arab & 3 & $(23.08)$ & & \\
\hline \multicolumn{5}{|l|}{ Marital status } \\
\hline Single & 7 & $(53.85)$ & & \\
\hline Married & 2 & $(15.38)$ & & \\
\hline Divorced & 4 & $(30.77)$ & & \\
\hline Number of children & & & 1.46 & 2.22 \\
\hline 0 & 6 & $(46.16)$ & & \\
\hline $1-2$ & 5 & $(38.46)$ & & \\
\hline $3+$ & 2 & $(15.38)$ & & \\
\hline \multicolumn{5}{|l|}{ Relationship } \\
\hline In a Relationship & 3 & $(23.08)$ & & \\
\hline Not in a Relationship & 10 & $(76.92)$ & & \\
\hline \multicolumn{5}{|l|}{ Religious faith } \\
\hline Religious & 1 & (7.69) & & \\
\hline Traditional & 5 & (38.46) & & \\
\hline Secular & 7 & $(53.85)$ & & \\
\hline
\end{tabular}

Socio-demographic characteristics of the participants as reported by self-report questionnaires. Note: $\mathrm{n}=13$

sexual behavior. Third, if the patient's condition deteriorated significantly during the active participation in the interview (CGI $\geq 5$ (. Notably, a relaxation protocol had been prepared in order to manage cases of restlessness following the interview. However, there was no need to use it.

\section{Research Tools}

The main research tool was a semi-structured interview. The interview addressed the patient's sexual knowledge and experiences, both in and outside the ward; the challenges of expressing his sexuality; and suggestions for streamlining the existing policy (see interview questions in Appendix A). In addition, according to the principles of qualitative research, nonverbal responses were also noted, and an investigator's diary was kept in which the interviewer documented insights and comments during and after the interviews. 


\section{Research Procedure}

The study was conducted at a psychiatric hospital in the southern district of Israel. It was approved by the directors of the ward and by the institutional review board (IRB). Psychiatric patients in the isolated wards were assessed by the medical staff to determine their ability to sign an informed consent and participate in the study. Pseudonyms were used throughout the paper in place of participants' real names.

In order to evaluate the severity of the patient's symptoms we used the Clinical Global Impression Scale, CGI [17]. The purpose of the questionnaire is to obtain information about the effect of the active psychotic process on sexual function while the participants are in a psychotic state. The interviewer rated the severity of the symptoms on a scale of 1 (normal, not ill) to 7 (very ill), based on all the medical information in her possession, relying on the opinions of the physician treating the subject and the nurse in charge of the ward or her deputy. Remission was assessed using the CGI questionnaire, as all participants were confirmed to have had partial remission with low CGI (3/4). Then, participants signed the informed consent form while the interviewer read the form to them and explained the research aims.

Later, participants completed the demographic questionnaire. In the case of difficulty in reading, the interviewer read the questions for them. After completing the questionnaire, an in-depth interview was conducted. Most interviews took place in a single session. However, longer interviews were sometimes divided into two sessions. All interviews were conducted face-to-face in Hebrew by the same interviewer, a female clinical psychologist and certified sex therapist, who works in the ward and had been familiar to participants prior to the interviews. Confidentiality was assured to each participant along with an explanation of the publication of his anonymous quotes. The interviews were conducted in an intimate environment, in the Mental Health Center. The patients were asked to agree to the recording of the interview. Interviews lasted an average of 45 min to an hour. During the interview, the interviewer attempted to establish trust and create a sense of ease. After the interviews, the patients' mental condition was assessed by the medical staff. Later, the interviews were translated into English by a native English speaker. The raw materials were given to the researchers without identifying details. The researchers were committed to maintaining the privacy of participants and all raw materials were kept in a safe place after the work had been completed.

Data Analysis All interviews were conducted by T.P.S, who is a $\mathrm{PhD}$ with academic training in qualitative research and certified sex therapist. All interviews were transcribed verbatim. The interviews were thematically analyzed. To enhance the trustworthiness of the study, the analysis process involved two independent raters, who were part of the research team and experienced in content analysis and qualitative research methods.

The analysis included three stages [14]. In the first stage, we conducted a holistic reading of each interview. In the second stage, the main themes were derived (through an analysis of each interview). In the third stage, the analysis focused on reorganizing the themes, finding sub-themes, identifying connections, and creating the final set of themes.

During the analysis (stage 2), the researchers conducted an initial evaluation of randomly selected $20 \%$ of the transcripts to assess interrater agreement. Unclear themes as well as cases of disagreement were discussed to reach consensus regarding the coding. Interrater agreement was measured through a percent agreement method [27]. The 
researchers reached a consensus on $84 \%$ of these items at this preliminary phase. This high consensus rate can be ascribed to the fact that both scholars are knowledgeable in the researched domain.

\section{Results}

In the interviews we identified two principal themes: challenges in realizing sexuality within the isolated ward and suggestions for improvements. The former theme was divided into several sub themes: performing sexual acts and exposure to sexuality within the ward; medications' side effects; dialogue with the staff; sexuality in the ward presented as crossing limits; and absence of a space in the facility to fulfill sexual needs. Below we present each of the themes and sub themes.

\section{Challenges in Realizing Sexuality}

\section{Performing Sexual Acts and Exposure to Sexuality Within the Ward}

The joint stay in the ward and the relationships that are often formed among patients sometimes produced sexual activity or exposure to sexual behavior of other patients. Thus, it seems that sexuality is manifested within the psychiatric institution. Participants' stay at the hospital provides opportunities for sexual acts with other patients or with oneself (and in some cases, intents or fantasies regarding the medical staff). Furthermore, patients are aware of other patients' sexual behavior, which may be uncomfortable for them. The closeness that comes from staying together in the ward creates opportunities for sexual contacts. For example, Nathan (35, Jewish) said:

I won't lie to you, I've had many cases of having sex in the wards throughout the years as part of the hospitalization, I don't know if I should open it up [...] There was a case that I had oral sex a few months ago with some guy in here and it was on the porch and then one of the patients saw and told the staff.

Nathan also described the explicit sexual proposals and insinuating looks from other patients:

People come to me and tell me, or there are all kinds of looks maybe in the shower and they come to me and tell me like, listen I've never experienced but um, I'm interested in trying.

These opportunities for same sex relations may cause a sense of harassment among some patients. Amir (38, Jewish) said:

Someone grabbed me and said, 'Can I kiss you? Only one, only one [kiss], please'. I told him, 'no, go away and leave me alone.

Another element to be taken into account is technology, and particularly the use of smartphones, which amplifies sexual expressions within the ward. Several interviewees mentioned that other patients, who access pornographic and other sexual content through their smartphones, raised sex related discourse in the ward. For example, Jamil (55, Arab) said: 
I'm using my smartphone and I'd talk with my wife a lot. I'd want to hear her voice. To tell her 'What are you doing now?' 'Touch your breasts, touch them', you know...

Through the telephone, and then calm down until I'd get back home".

\section{Medication Side Effects}

Most of the patients suffer from side effects due to medication that interferes with their function in general and their sexual function in particular, affecting them physiologically and emotionally. This reality seems to have caused many of them to have negative attitudes towards medication.

Danny (47, Jewish) described how medication has diminished his sexual desire:

Firstly, it's suppressing, there is no point, no desire. This is a depressive reaction to the medication. Secondly, it's hurting my desire, my will, the way in which my desire is expressed.

Furthermore, some patients indicated specific physiological damage to their sexual functioning, such as erectile dysfunction and premature ejaculation. According to Yosef (43, Jewish), for example:

I want to have sex 3-4 times, and I can't because I don't have a good erection.

Amir (38, Jewish), in contrast, presented a different perspective, detailing how the medication actually helped his sexual functioning:

Solian (Amisulpride, medication for treating schizophrenia) bothered me. Rispond (Risperidone, a different drug for schizophrenia) helped me, I did so much sex I almost fainted.

There appears to be an additional price that participants pay for their interrupted sexual function, which is the concern they feel as men experiencing sexual difficulties.

Some patients also indicated serious harm caused by the medications' side effects, including decreased self-esteem and a diminished sense of masculinity and control. As Jamil (55, Arab) put it:

When I'm visiting my wife, around my wife I feel myself worthless. In terms of manhood.

\section{Dialogue with the Staff}

The interviews revealed different views on the availability of the therapeutic staff in the dialogue on sexuality and the complexity of such dialogue. Not all patients were aware of the possibility of discussing sexuality with the staff. Moreover, many wondered if raising the subject with a staff member might be useful, worrying about the responses they might get.

According to Sammy (40, Jewish):

I can talk to the doctor. Maybe she'll get this conversation and maybe not, maybe this isn't the place at all. Right?

Herzl (45, Jewish) expressed the feeling that there is nobody to talk to in the ward regarding sexual issues. 
To the walls. The walls. Nobody talks about it.

Yet, other patients expressed no difficulty in raising the topic with staff members. As Nathan (35, Jewish) put it:

I've heard people talking about it with the staff with no problem.

When the interviewees were asked about the reasons sexual issues are not discussed with the staff, they mentioned lack of interest or low availability as the main reasons. According to Danny (47, Jewish):

It's because nobody cares. The doctor, psychiatrist, he asks other things entirely, like 'Are you hearing voices?' [...] There is only one doctor for twelve patients, and he may talk to us every day but he doesn't really have the time to listen.

\section{Sexuality in the Ward Presented as Crossing Limits}

Many patients feel that expressing sexual content during their stay in the ward is inappropriate. Some feel that practicing sexuality is presented in the department as crossing limits.

Several patients share experiences of "getting caught" having sex or masturbating, which was either ignored by the staff or provoked an angry, punitive response. Haled (61, Arab) said:

It's not the right place to masturbate. It's a hospital, you need to respect the hospital.

Nathan (35, Jewish) says that the staff's attitude towards sexuality makes the patients see it as taboo, and fear relating to it:

Really maybe if there was a little bit more reference to the topic [...] would be like healthy to this topic, so maybe it would light things up more positively, rather than being such a taboo.

In his interview, Nathan said that the doctor had instructed him to avoid expressing his sexual needs:

There was a doctor that was the director of some ward, who told me that I think I am an actor in an erotic movie and he stopped me from talking about sexuality in the ward. He asked me to stop looking for it.

\section{Absence of a Space in the Facility to Fulfill Sexual Needs}

Patients find it difficult to find an appropriate place to realize sexuality in the ward. They describe the need for sexuality as a source for discomfort and concealment. Many interviewees described the poor conditions in the ward to fulfill their sexual needs. For example, Benny (36, Jewish) says:

I can fulfill my sexuality only if it feels intimate enough for me, for example in the shower or under the blanket when everyone was sleep.

In light of this reality, some patients choose to entirely avoid sexual acts within the ward, as Haled (61, Arab) indicates:

Nowadays I don't have sex because I'm in the psychiatric hospital. [...]Outside [of the hospital], yes. But here, no. I don't have any privacy here. 
Danny (47, Jewish) mentioned the existence of cameras (placed in the ward for security reasons) and protested the current policy, which does not designate special rooms to serve patients' sexual needs:

Conjugal visits are legislated by the law. Our human rights are violated, compared to inmates in prison. If I'm here, I don't even have the option to have a conjugal visit with my partner, it's out of the question. It's unbelievable. Even if I had asked, there is no place. Will they provide us a private room? And will they allow us to get inside alone? They'll put cameras inside [...] it's something basic that human beings need like a toilet, that's the same thing, that's hard.

In contrast, some patients presented masturbation within the ward and finding a place to perform it as a routine manner, which did not seem to cause them any difficulty or embarrassment. For example, according to Herzl (45, Jewish):

Nothing can stop me. I go to the toilet, I make sure nobody's around. I've been here one month, and I did it at least ten times since I came. No problem.

\section{Suggestions for Improvements in the Hospital}

Evidently, the patients were aware of the existing situation and had hopes for improvements in their life quality. They proposed several policy changes that would provide solutions to the problems that were raised. Additional ideas for improvements stem from the patients' experiences as reflected in the interviews.

One of the patients' main proposals was starting an open dialogue with the staff about sexuality, either in personal sessions or in group discussions. Patients believe that such discourse would be helpful to their stay in the ward. According to Sammy (40, Jewish), for example, the patients would be interested in such dialogue and the staff must initiate it:

You need to create a group to talk about sexuality. And then to hear, if a person speaks correctly, to encourage them to keep going that way.

Yet to allow a meaningful dialogue on sexuality with the medical staff, the right conditions must be set up. The interviews indicate several important points that might help the patients talk about their sexuality and promote effective future dialogue. First, the gender of the caregiver is important. Yossi (43, Jewish), for example, states that he shared his problem of sexual dysfunction with his male psychiatrist since he felt comfortable talking to him:

Interviewer: "And you felt comfortable talking to the psychiatrist about it?"

Yossi: "Yes, because my psychiatrist was a man".

Second, the medical staff should approach the subject in a manner that suits each of the patients, depending on their preferences and uniqueness. As some interviewees mentioned, they are reluctant to participate in group sessions. For example, Benny (36, Jewish) said:

It's intimate, you don't get into it in group sessions.

Third, the patient should be the one deciding if and whom to confide in. The decision might be based on a personal connection with a certain staff member or their professional role in the ward. As Benny (36, Jewish) said: 
A doctor shouldn't get into the intimate world of the patient, that's what you have psychologists for.

Fourth, staff members must be empathic so that they can handle the emotionally charged issue of sexual dysfunction or any such intimate problem. Interviewees also mentioned their thirst for relevant knowledge about sexuality. According to Herzl (45, Jewish):

The doctors have to give them this kind of information.

In addition, most interviewees mentioned the need for a private room, free of cameras, to serve patients' needs. As Danny (47, Jewish) said:

If they let me [decide], I'd made a room for conjugal visits and I'd give an option for a closed visit for couples, they need that option. If someone has a partner, he should have an option for a conjugal visit. And even more, there should be an option for a private place and a conjugal visit with as many partners as you wish.

As part of the supervision and regulation of this issue, measures for safe sex should be supplied to patients, as we can understand from Sammy's statement (40, Jewish):

One of the reasons that we don't have sex is because there isn't any protection.

A contrasting opinion was that of Amir (38, Jewish) who, when asked about suggestions for improving the current reality, suggested avoiding a discussion of sexuality altogether. This represents an alternative view, of patients who might feel uncomfortable dealing with matters of sexuality and prefer to focus on other aspects of healing and rehabilitation.

\section{Discussion}

The current study examined sexuality and its manifestations among hospitalized psychiatric patients. The findings indicate a gap between the existence of sexual needs and the absence of an actual response from the therapeutic staff and policymakers. The interviews presented various challenges that the patients are facing, such as exposure to other patients' sexual behavior in a manner that causes discomfort, medication side effects that bear on sexuality, lack of open discourse with the staff, sexuality presented in the department as crossing limits, and lack of space for sexual needs fulfillment. These challenges can potentially interfere with the patients' right for healthy sexuality, as the patients themselves pointed out.

Moreover, the interviews brought up possible solutions that emerged from the patients' experiences. They proposed concrete practical solutions (such as allocating rooms for conjugal visits or private sex activity) as well as solutions relating to legitimizing patients' sexuality and developing various ways to enhance open and effective sexuality-related dialogue between patients and staff.

[35] found that approximately 15-30\% of psychiatric patients reported having sexual contact during their hospitalization. They also found that over $67 \%$ of the patients experienced sexual dysfunction. Psychiatric inpatients face special difficulties such as stigmatization and concern of the staff about their judgment and ability to consent to sexual intercourse. In turn, other studies have shown that addressing the sexuality of patients contributes to their mental well-being and reduces risk behaviors [3, 8, 12, 15, 19, 26].

From the interviews we learned that the patients had acute sexual awareness, clear perceptions, and sexual experiences. The centrality of the subject in their lives could not be 
doubted. Yet, in accordance with Cort et al. [13], the present investigation demonstrates that there still exists much work to be done. Although patients sometimes feel that they can discuss their sexual needs with the staff, it is clear from the interviews that in general, the staff does not actively address the topic. There is also no evidence of a systematic intervention program currently taking place in the department.

According to Buckley et al. [11], whether sexual relations are permitted within the departments or not, sexuality will exist and be expressed among psychiatric patients. The current study confirms this finding. The interviewees indicated that they were exposed to other patients' sexuality and engaged in sexual activity themselves, although sexual intercourse is considered unacceptable in the department. According to Shalev et al. [35], hospitalization includes many aspects of sexual behavior. The regulations regarding what is permitted and what is taboo are not always clear to the patients, which creates a feeling of discomfort.

Conducted among men in an isolated ward, the present study reinforces the existing body of knowledge [7], conforming the medicinal side effects that influence sexual functioning, and provides additional qualitative information on the psychological and social effects of this functional impairment. The literature proposes solutions to medication side effects. For example, according to Balon [4], physicians should manage the topic appropriately throughout the patient's initial assessment and prior to the administration of medication, including a discussion with the patient about the potential harm to his sexual functioning and possible coping strategies.

Cook [12] addressed the absence of privacy in psychiatric facilities, which also surfaced in this study as a significant challenge. Patients resort to acting in hiding and finding alternatives for fulfilling their sexuality. Based on interviewees' narratives, it is recommended to adopt the following changes: first, the most obviously needed step is to form an open dialogue on this topic, whether in a group or an individual setting, providing knowledge and guidance to patients about biological and psychological aspects of sexuality. Since the topic is intimate and sensitive and might lead to uncomfortable feelings among both the patients and the staff, the dialogue should be appropriate and conducted carefully. Moreover, the staff should be properly trained, so that its members become aware of patients' sexual needs, and consequently adopt a positive attitude toward patients' sexuality, legitimize and normalize it.

In addition, a private room should be allocated for respectful implementation of sexual needs and conjugal visits, which will protect the patients' safety and improve the lives of patients and staff. Realization of sexuality in the department will be possible only with the support of the staff and the management, whose role is to enable sexuality, set limits, encourage dialogue on the topic, and provide suitable space and means. We call for giving space to the sexual needs of patients in a controlled manner, with restrictions and clear boundaries that will preserve the dignity and safety of patients. The medical staff should acknowledge that patients have one limitation - their mental illness, but otherwise, they are people with healthy human needs.

The current study enriches existing knowledge and provides a broad picture of patients' experiences. We are not aware of any studies that have been done on male patients with SMI who are hospitalized in an isolated ward. This study is also unique in its target population, which is comprised of male patients in a closed ward who come from diverse cultures and from a region in Israel considered as geographic and social periphery. The findings indicate that sexuality crosses cultures, ethnicities, and social status. It is a basic human need [20], as reflected in the interviews conducted with this unique population. The study allows us to examine the sexual needs of a conservative population that differs from the 
populations studied thus far in Europe and the United States. The study may help in mapping participants' sexual needs, gaps, and challenges, and in understanding the adaptive and non-adaptive coping methods assumed by the patients.

Finally, the research may lead to policy changes and the formulation and implementation of an intervention program that includes psycho-educational information about healthy sexuality. Such a program, based on proper communication and setting of boundaries, will have implications for therapeutic interventions. Addressing patients' sexuality in this manner will enable it to be channeled into healthy places.

The study has several limitations. First, the fact that some of the participants found it difficult to discuss the topic of sexuality may bias the results. Second, although according to the preliminary assessment, the interviewees were qualified in terms of their mental stability to participate in the study, in practice, it was difficult to maintain attention throughout the interview and to retain continuity and coherence during all interviews. For this reason, some of the interviews were split when needed. Yet, the interviewer was a clinical psychologist who is trained in the treatment of psychiatric inpatients. An additional limitation stems from the fact that the interviewer was a woman while the interviewees were men, leading to possible bias in the results. Since the study population consisted of men in a closed ward, care must be taken when generalizing the findings elsewhere. Future studies should be conducted in other populations, such as female patients, ambulatory psychiatric patients, and medical personnel. Another limitation might be that the participants knew the interviewer. However, it should be noted that she wasn't their case manager therapist.

\section{Conclusions}

There is no doubt that sexuality exists and is prominent in the lives of patients in the isolated psychiatric ward. The path towards an integrative solution to this issue is still long and requires courageous coping of the patients themselves, the staff, and policymakers. Despite the growing awareness among the public and the caregivers, it is evident that this issue is not usually discussed openly. Recognizing its importance and integrating it into the daily therapeutic agenda in an open and honest manner might lead to creative solutions for the above-mentioned challenges. Realizing that the main rift today is between the understanding that the patients have the right for sexual expression and the actual implementation of this right, we emphasize the importance of changing the policy to create a new agenda. Our findings emphasize the need to address the issue of sexuality among hospitalized psychiatric patients, while listening to their voices and helping them in the process of rehabilitation and recovery.

\section{Appendix A: Study protocols}

See Table 2. 
Table 2 The study protocols

First protocol guide

What used to be the meaning of sexuality for you?

What have you heard about sexuality from other people?

What sexual experiences did you have before coming to the hospital?

What are your current sexual preferences?

What are the difficulties and challenges you encounter when trying to fulfil your sexuality?

What helps you express your sexuality in an enjoyable way?

How do you think your mental illness affects your sexuality?

How does your hospitalization affect your sexuality?

How do the medications affect your sexuality?

Who do you talk to about your sexuality now? At the hospital? Outside the hospital?

Do you have any suggestions for how to change things at the hospital so that you and people like you are better supported in a way that makes you sexually healthier?

Second protocol guide

In what ways can you express your sexuality in the psychiatric ward?

How do you engage in new relationships inside the ward? How do you maintain relationships from the time you were not hospitalized?

How do you think the staff apprehend sexuality of the patients?

How do you cope with the ward restrictions when you want to express your own sexuality?

Have you been exposed to sexual abuse or harassment inside the ward? By whom? Did you report it to the staff?

Authors' Contribution MK and TP-S contributed equally to the research and the manuscript. JG and IS analyzed the interviews. Manuscript was written by the four authors.

Funding No funding.

\section{Declarations}

Conflict of interest The authors declare that they have no conflict of interest.

Open Access This article is licensed under a Creative Commons Attribution 4.0 International License, which permits use, sharing, adaptation, distribution and reproduction in any medium or format, as long as you give appropriate credit to the original author(s) and the source, provide a link to the Creative Commons licence, and indicate if changes were made. The images or other third party material in this article are included in the article's Creative Commons licence, unless indicated otherwise in a credit line to the material. If material is not included in the article's Creative Commons licence and your intended use is not permitted by statutory regulation or exceeds the permitted use, you will need to obtain permission directly from the copyright holder. To view a copy of this licence, visit http://creativecommons.org/licenses/by/4.0/.

\section{References}

1. Abu-Bader, S., Gottlieb, D.: Poverty, education and employment in the Arab-Bedouin society: a comparative view. National Insurance Institute Research Fund [Hebrew] (2009)

2. Al-Krenawi, A., Graham, J.R., Al-Bedah, E.A., Kadri, H.M., Sehwail, M.A.: Cross-national comparison of Middle Eastern university students: help-seeking behaviors, attitudes toward helping professionals, and cultural beliefs about mental health problems. Commun. Ment. Health J. 45(1), 26-36 (2009) 
3. Assalian, P., Fraser, R., Tempier, R., Cohen, D.: Sexuality and quality of life of patients with schizophrenia. Int. J. Psych. Clin. Pract. 4(1), 29-33 (2000)

4. Balon, R.: SSRI-associated sexual dysfunction. Am. J. Psych. 163(9), 1504-1509 (2006)

5. Barnes, D., Boland, B., Linhart, K., Wilson, K.: Personalisation and social care assessment-the care act 2014. BJPsych. Bull. 41(3), 176-180 (2017)

6. Bergman-Levy, T., Haklai, Z., Yoffe, R., Pugachova, I., Goldberger, N., Aburbe, M.: Mental Health in Israel-Statistical Abstract 2019. State of Israel, Ministry of Health, Mental Health Division and Health Information Division, Jerusalem (2021)

7. Bobes, J., Garcia-Portilla, M.P., Rejas, J., HernNdez, G., Garcia-Garcia, M., Rico-Villademoros, F., Porras, A.: Frequency of sexual dysfunction and other reproductive side effects in patients with schizophrenia treated with risperidone, olanzapine, quetiapine, or haloperidol: the results of the EIRE study. J. Sex Marital. Ther. 29(2), 125-147 (2003)

8. Brown, S.D., Reavey, P., Kanyeredzi, A., Batty, R.: Transformations of self and sexuality: psychologically modified experiences in the context of forensic mental health. Health 18(3), 240-260 (2014)

9. Buckley, P.F., Hyde, J.L.: State hospitals' responses to the sexual behavior of psychiatric inpatients. Psych. Serv. 48, 398-399 (1997)

10. Buckley, P.F., Robben, T.: A content analysis of state hospital policies on sex between inpatients. Psych. Serv. 51, 243-245 (2000)

11. Buckley, P.F., Robben, T., Friedman, L., Hyde, J.: Sexual behavior in persons with serious mental illness (Patterns and clinical correlates) (1-20). In: P.F. Buckley (Ed.) Sexuality and Serious Mental Illness. Gordan \& Breach Publications (1999)

12. Cook, J.A.: Sexuality and people with psychiatric disabilities. Sex. Disabil. 18(3), 195-206 (2000)

13. Cort, E.M., Attenborough, J., Watson, J.P.: An initial exploration of community mental health nurses' attitudes to and experience of sexuality related issues in their work with people experiencing mental health problems. J. Psychiatr. Ment. Health Nurs. 8, 489-499 (2001)

14. Creswell, J.W., Poth, C.N.: Qualitative Inquiry and Research Design: Choosing Among Five Traditions. Sage Publications Inc, Thousand Oaks (2017)

15. Deegan, P.: Human sexuality and mental illness: Consumer viewpoints and recovery principles. In: Buckley, P.F. (ed.) Sexuality and Serious Mental Illness, pp. 21-33. Gordan \& Breach Publications (2001)

16. Del Mar Baños-Martín, M., Márquez-Hernández, V.V., Gutiérrez-Puertas, L., Aguilera-Manrique, G., Gutiérrez-Puertas, V., Granados-Gámez, G.: Sexual behavior in patients with psychosis admitted to a hospital unit. Issues Ment. Health Nurs. 38(6), 473-479 (2017)

17. Guy, W.: Clinical Global Impression (CGI) Scale. ECDEU Assessment Manual for Psychopharmacology-Revised. Rockville, Maryland: US Department of Health, Education, and Welfare. Public Health Service, Alcohol, Drug Abuse, and Mental Health Administration, NIMH Psychopharmacology Research Branch, Division of Extramural Research Programs (1976)

18. Gruskin, S., Yadav, V., Castellanos-Usigli, A., Khizanishvili, G., Kismodi, E.: Sexual health, sexual rights and sexual pleasure: meaningfully engaging the perfect triangle. Sex. Reprod. Health Matter. 27(1), 29-40 (2019)

19. Higgins, A., Barker, P., Begley, C.M.: Sexuality and mental health nursing in Ireland: weaving the veil of socialized inhibition. Nurse Educ. Today 29(3), 357-364 (2009)

20. Incedere, A., Küçük, L.: Sexual life and associated factors in psychiatric patients. Sex. Disabil. 35(1), 89-106 (2017)

21. Kaplan, H., Najjar, Z., Kalnisky, E., Keinan, A.: The challenge of diversity in teacher education institutions: students' sense of relatedness and perceptions regarding being a minority or majority. J. Diver. Higher Educ. Advance online publication

22. Kismödi, E., Corona, E., Maticka-Tyndale, E., Rubio-Aurioles, E., Coleman, E.: Sexual rights as human rights: a guide for the WAS declaration of sexual rights. Int. J. Sex. Health 29(sup1), 1-92 (2017)

23. Levaot, Y., Meiri, G., Dinstein, I., Menashe, I., Shoham-Vardi, I.: Autism Prevalence and Severity in Bedouin-Arab and Jewish Communities in Southern Israel. Commun. Mental Health J. 55(1)

24. Maslow, A., Lewis, K.J.: Maslow's hierarchy of needs. Salenger Incorp. 14(17), 987-990 (1987)

25. Maylea, C.: The capacity to consent to sex in mental health inpatient units. Aust. N. Z. J. Psychiatry 53(11), 1070-1079 (2019)

26. McClure, L.: Where is the sex in mental health practice? A discussion of sexuality care needs of mental health clients. J. Ethics Mental Health 7, 1-6 (2012)

27. McHugh, M.L.: Interrater reliability: the Kappa statistics. Biochemia Medica 22(3), 276-282 (2012)

28. Mossman, D., Perlin, M.L., Dorfman, D.A.: Sex on the wards: Conundra for clinicians. J. Am. Acad. Psych. Law 25, 441-460 (1997) 
29. National Institute of Mental Health (2019). https://www.nimh.nih.gov/health/statistics/mental-illness. shtml

30. Omnibus Budget Reconciliation Act of 1987. Section 4201, 42 U.S.C.A. Section 1396r(c)(3) (C) (West 1992). https://archive.org/stream/omnibusbudgetrec00unit_16/omnibusbudgetrec00unit_16_djvu.txt

31. Paul-Binyamin, I., Haj-Yehia, K.: Multicultural education in teacher education: Shared experience and awareness of power relations as a prerequisite for conflictual identities dialogue in Israel. Teach. Teach. Educ. 85, 249-259 (2019)

32. Ravenhill, J.P., Poole, J., Brown, S.D., Reavey, P.: Sexuality, risk, and organisational misbehaviour in a secure mental healthcare facility in England. Cult. Health Sex. 22(12), 1382-1397 (2020)

33. Rennie, C.: The construction of sex and sexuality within clinical psychology training and practice. Canterbury Christ Church University (2018)

34. Sathasivam, R., Vidanapathirana, M.: Sexual rights of the psychiatric patients, consenting capacity and contradiction with local and national policies and the law of Sri Lanka: a case report. Medico-Legal J. Sri Lanka 9(1) (2021)

35. Shalev, A., Weil, G., Rubinstein, L.: Sexuality in psychiatric hospitalization: reality versus policy. Medicine, 155(12), 762-766 (2016)

36. Substance Abuse and Mental Health Services Administration. Key substance use and mental health indicators in the United States: Results from the 2019 National Survey on Drug Use and Health (HHS Publication No. PEP20-07-01-001). Rockville, MD: Center for Behavioral Health Statistics and Quality, Substance Abuse and Mental Health Services Administration (2020). https://www.samhsa.gov/ data/sites/default/files/reports/rpt29393/2019NSDUHFFRPDFWHTML/2019NSDUHFFR1PDFW090 120.pdf

37. Swirski, S., Konor-Attias, E., Lieberman, A.: Israel: a social report 2017. Adva center - Information on Equality and Social Justice in Israel. Retrieved on 21.8.19 from: http://adva.org/wp-content/uploads/ 2018/02/Social-Report-2017.pdf [Hebrew]

38. Tennille, J., Wright, E.: Addressing the intimacy interests of people with mental health conditions: acknowledging consumer desires, provider discomforts, and system denial. A monograph from the Temple University collaborative on community inclusion of individuals with psychiatric disability (2013). http://tucollaborative.org/sdm_downloads/addressing-the-intimacy-interests-of-people-withmental-health-conditions-acknowledging-consumer-desires-provider-discomforts-and-system-denial

39. Tiwana, R., McDonald, S., Vollm, B.: Policies on sexual expression in forensic psychiatric settings in different European countries. Int. J. Ment. Heal. Syst. 10(1), 1-11 (2016)

40. World Health Organization, Defining sexual health: Report of a technical consultation on sexual health (2016)

Publisher's Note Springer Nature remains neutral with regard to jurisdictional claims in published maps and institutional affiliations.

\section{Authors and Affiliations}

\section{Michal Kaplan ${ }^{1}$ - Tal Peleg-Sagy ${ }^{2,3}$ • Jonathan Guez ${ }^{2,4} \cdot$ Iftach Sagy ${ }^{3,5}$}

Tal Peleg-Sagy

neshikotal@hotmail.com

Jonathan Guez

jonjon@bgu.ac.il

Iftach Sagy

iftachsagy@gmail.com

1 Ben Gurion University of the Negev, Yeelim 3, Meitar 8502500, Beer-Sheva, Israel

2 Beer-Sheva Mental Health Center, Beer-Sheva, Israel

3 Ben Gurion University of the Negev, Beer Sheva, Israel

4 Department of Psychology, Achva Academic College, Beer-Tuvia, Israel

5 Clinical Research Center, Soroka University Medical Center, Beer Sheva, Israel 